\title{
MODELAGEM MATEMÁTICA PARA APLICAÇÃO DE SISTEMAS DE WETLANDS NO TRATAMENTO DE AQÜÍFEROS LIVRES E RASOS CONTAMINADOS
}

\author{
MATHEMATICAL MODELING APPLIED TO TREATMENT \\ OF CONTAMINATED SHALLOW AQUIFERS USING WETLANDS
}

\author{
Jeane G. Santos ${ }^{1}$ Ricardo Hirata ${ }^{1}$ e Luiz C. K. M. Ferrari ${ }^{1}$
}

Recebido em:03/06/2005, aceito em:30/08/2006

\begin{abstract}
RESUMO
Wetlands são habitats cujo nível de água encontra-se imediatamente abaixo, acima ou ao nível do solo. Exemplos de wetlands naturais são pântanos e brejos. Há também as artificiais, geralmente construídas com propósitos ambientais. Quatro possíveis cenários de aplicação de wetlands na atenuação de plumas contaminantes em aquíferos rasos, sem auxílio de bombeamento, foram criados utilizando-se o software Visual Modflow. Três cenários semelhantes, representando sistemas de fluxo superficial, com variação na geometria da janela de entrada de água, na profundidade de captura da pluma de contaminação e na recarga, foram comparados entre si. Os resultados mostraram que o volume de água que atravessa a wetland varia inversamente com o tempo de residência da água no canal e ambos são controlados pela condutividade hidráulica do aqǘfero. Em simulações com recarga de $250 \mathrm{~mm} / \mathrm{ano}$, os tempos foram menores que aqueles obtidos nas simulações sem recarga. A dimensão horizontal da pluma capturada aumentou com a construção de barreiras na forma de funil, usadas para alargar a abertura permeável da wetland. Já a inserção de barreiras verticais, de até $4 \mathrm{~m}$ abaixo da abertura permeável, para aumentar a profundidade de captura, não se mostrou efetiva. Um quarto modelo, representando uma wetland de fluxo superficial seguido de um sistema de fluxo subsuperficial, necessitou de uma área de $2000 \mathrm{~m}^{2}$ para tratar apenas $16 \mathrm{~m}^{3} /$ dia. Os resultados apontaram a viabilidade do uso do Visual Modflow para auxiliar na criação de wetlands. Porém, a utilização desses sistemas para capturar água subterrânea sem o auxílio de bombeamento foi pouco efetiva.
\end{abstract}

Palavras chave: Wetlands, Modelagem Matemática, Aqüíferos Rasos

\begin{abstract}
Wetlands are habitats where water level can be either above, beneath or at surface ground. Swamp and marsh are examples of natural wetlands. However, there are also the manmade ones, generally constructed for environmental purposes. Using Visual Modflow were created four possible wetlands application scenarios for the contaminant plume attenuation in shallow aquifers, without pumping (natural gradient). Three of them, representing superficial flow systems, were compared one each other, varying window water entrance geometry and depth in the contaminant plume capture and recharge. Results indicate that water volume that enters into the wetland varies inversely with the water residence time in the channel and both are controlled by aquifer hydraulic conductivity. In simulations using $250 \mathrm{~mm} / \mathrm{y}$ of recharge, the residence time was smaller than the ones obtained with no recharge simulations. The horizontal dimension of captured plume can be enlarged with the building up of an impermeable funnel barrier in the entrance of the wetland, used to enlarge wetland permeable entrance. However, inserting vertical barriers, at least $4 \mathrm{~m}$ below the wetland entrance, the results were not so effective. A forth model, representing a shallow wetland followed by a shallow flow system, required a $200 \mathrm{~m}^{2}$ area to treat $16 \mathrm{~m}^{3} /$ day. Modeling results indicated viability of groundwater treatment using wetlands, although, large areas are required to have an effective groundwater plume remediation.
\end{abstract}

Keywords: wetlands, mathematical modeling, shallow aquifer

\section{INTRODUÇÃO}

Aqǘferos rasos são extremamente suscetíveis à contaminação, tanto por compostos orgânicos quanto por microorganismos patogênicos. Isto ocorre devido à zona nãosaturada, incluindo a camada de solo, ser pouco espessa, diminuindo a capacidade de atenuação de contaminantes.

Um dos métodos utilizados, principalmente em países desenvolvidos, para o tratamento de águas contaminadas é o sistema de wetlands, tanto para a água subterrânea quanto a água superficial. Este tipo de habitat pode ocorrer naturalmente ou ser criado artificialmente, e caracteriza-se por ecossistemas que ficam parcial ou totalmente inundados durante pelo menos algum período do ano. Suas características e propriedades variam conforme a geologia, a geomorfologia e as condições climáticas da área considerada.

Wetlands artificiais têm sido largamente empregadas na remediação de águas superficiais contaminadas, sendo ainda pouco avaliada em casos de atenuação de plumas contaminantes em águas subterrâneas. Nestes casos a água é normalmente bombeada para dentro de um lago, onde o ambiente químico gerado, juntamente com plantas aquáticas comuns para esses sistemas, promove a remoção dos contaminantes, principalmente por processos de transformação química e adsorsão.

Devido ao baixo custo de operação e à facilidade de implantação, a hipótese de utilização de sistemas de wetlands artificiais no tratamento de água subterrânea insinua-se como

${ }^{1}$ Departamento de Geologia Sedimentar e Ambiental - Instituto de Geociências da Universidade de São Paulo (j.glaucia@uol.com.br); (rhirata@usp.br);(luiz.ferrari@modcom.com.br) 
uma alternativa interessante (ainda não devidamente estudada), principalmente em áreas habitadas por população de baixa renda, que eventualmente utiliza fossas negras e sépticas para eliminação do seu esgoto.

$\mathrm{O}$ primeiro sistema de wetland artificial utilizado para tratamento de água superficial no Brasil foi construído por SALATI et al. (1982 in SALATI et al., 1998), próximo ao rio Piracicamirim, no município de Piracicaba (SP). Desde então, novas tecnologias vêm sendo desenvolvidas na tentativa de aumentar a eficiência das wetlands e reduzir os investimentos na despoluição de águas.

Alguns autores utilizaram a modelagem matemática na verificação da interação de wetlands naturais e artificiais com a água subterrânea (HENSEL; MILLER, 1991; BRADLEY, 1996, in RESTREPO et al., 1998). Neste trabalho testou-se a possibilidade de empregarem-se wetlands construídas na remoção de contaminantes de aqüíferos rasos livres, sem o auxílio de bombeamento, calculadas por diferentes geometrias de modelos numéricos.

A equação de água subterrânea relacionada a cada um dos modelos foi resolvida com o uso do programa computacional Visual Modflow da Waterloo Hydrogeologic Inc. (GUIGUER; FRANZ, 1996), para um regime estacionário de fluxo, obtendo-se assim a distribuição de cargas e respectivo fluxo de água subterrânea na região modelada.

\section{MODELOS MATEMÁTICOS CRIADOS Descrição dos Modelos}

Inicialmente, foram produzidos três modelos básicos distintos M1, M2, M3. Uma vez que esses modelos não apresentaram eficiência satisfatória, construiu-se posteriormente o modelo M4, cuja geometria foi ajustada com o objetivo de atingir o melhor resultado dentro de certos limites imposto, ou seja vazões de saída do sistema superiores a $10 \mathrm{~m}^{3} / \mathrm{dia}$ e tempo de residência da água dentro do sistema ente 10 e 15 dias (tempo observado em algumas wetlands artificiais existentes).

Os modelos criados simularam a porção $P$ de um aqüífero, cujas dimensões nas coordenadas $X$ (largura), Y (comprimento) e Z (profundidade), e os números de linhas, colunas e camadas matemáticas (layers) estão descritos na Tabela 1 e representados nas Figuras 1 e 2.

A eficiência dos modelos M1, M2 e M3, comparados entre si (o modelo M4 foi analisado isoladamente), na captura da água subterrânea e sua permanência dentro do sistema foram medidas através dos fatores:

- $\quad t_{\text {trânsito }}$ - o tempo que a água permanece no interior do sistema (dias);

- $\quad \mathrm{P}_{\text {captura }}$ - a maior profundidade que um tubo de fluxo que penetra o sistema pode atingir antes de ser capturado (m);

- $\quad \mathrm{L}_{\text {captura }}$ - a maior largura de captura de uma pluma que a wetland consegue capturar (m);

- $\mathrm{Q}_{\text {dreno }}$ - a vazão da água que deixa a cavidade após ter permanecido nela por um intervalo de tempo suficiente para a remoção da contaminação ( $\mathrm{m}^{3} /$ dia).

Esses parâmetros foram obtidos com a inserção de partículas nas aberturas permeáveis das cavidades e pelo balanço de massa calculado pelo módulo ZBUD do Visual Modflow.

As partículas caminham por advecção e agem como traçadores do fluxo de água subterrânea, sendo possível, através das suas trajetórias, determinarem a geometria dos tubos de fluxo.

Devido às limitações do programa, o interior de cada canal foi definido como uma região de condutividade hidráulica de $1 \mathrm{~m} / \mathrm{s}$ e porosidade de 0,95 (KADLEC; KNIGHT, 1996). O material do volume $P$ (aqüífero), tomado como homogêneo e isotrópico quanto às propriedades hidráulicas, foi associado a uma porosidade efetiva de 0,15 e a uma condutividade hidráulica $\mathrm{K}_{\text {terreno, variável. }}$

$\mathrm{Na}$ região de saída da água da cavidade estabeleceu-se um dreno $D$ de alta condutância (1000 $\mathrm{m}^{2} /$ dia), a uma altura $H$, variável em relação à base de $P$, escolhida de modo a manter uma coluna de água $\left(\mathrm{H}_{\text {dreno }}\right)$ entre $0,3 \mathrm{~m}$ e $1 \mathrm{~m}$ no interior da cavidade, condição necessária para manter as plantas ali existentes (KADLEC; KNIGHT, 1996). Ao fundo e nas paredes de cada canal estabeleceu-se uma baixa condutividade hidráulica $\left(1 \times 10^{-10} \mathrm{~m} / \mathrm{s}\right)$, exceto em uma pequena janela $J$ de $10 \mathrm{~m}$ de largura, para condicionar o trajeto da água para dentro da cavidade.

O modelo M1 (Figura 3) foi construído de acordo com as características gerais de uma wetland de fluxo superficial. 
Tabela 1 - Características geométricas da porção $P$ do aquiífero para cada modelo (M)

Table 1- Geometry of the portion $P$ of the aquifer for " $M$ " model

\begin{tabular}{c|c|c|c|c|c}
\hline \multicolumn{2}{c|}{ Modelo } & M1 & M2 & M3 & M4 \\
\hline \multirow{3}{*}{ Região $R$} & $\mathbf{X}(\mathbf{m})$ & 30 & 30 & 30 & 50 \\
\cline { 2 - 6 } & $\mathbf{Y}(\mathbf{m})$ & 100 & 100 & 100 & 100 \\
\cline { 2 - 6 } & $\mathbf{Z}(\mathbf{m})$ & $20-21$ & $20-21$ & $20-21$ & $20-22$ \\
\hline Linhas & 100 & 100 & 100 & 100 \\
\hline Colunas & 30 & 30 & 30 & 50 \\
\hline Camadas Verticais & 22 & 22 & 22 & 17 \\
\hline
\end{tabular}

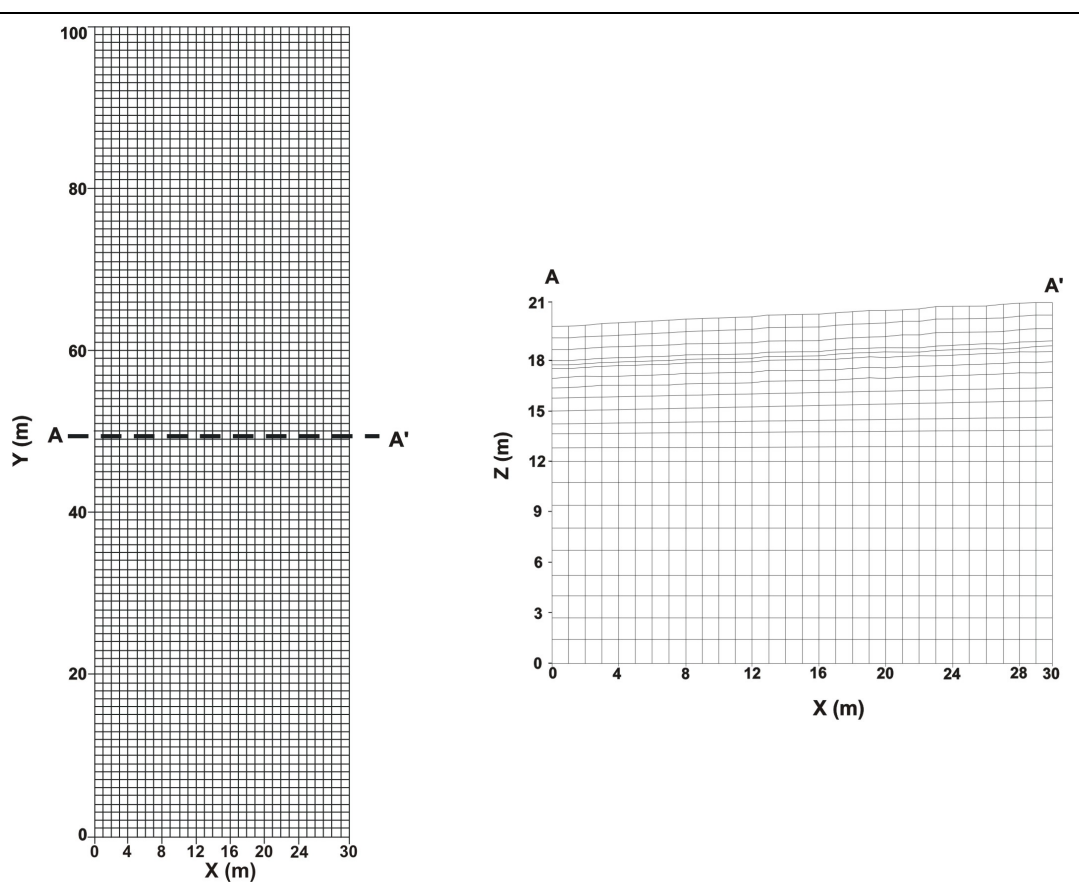

Figura 1- Geometria da porção $P$ dos modelos M1, M2 e M3 (planta e perfil A-A')

Figure 1- Geometry of portion P of the M1, M2 and M3 models (plan view and vertical profile A-A')

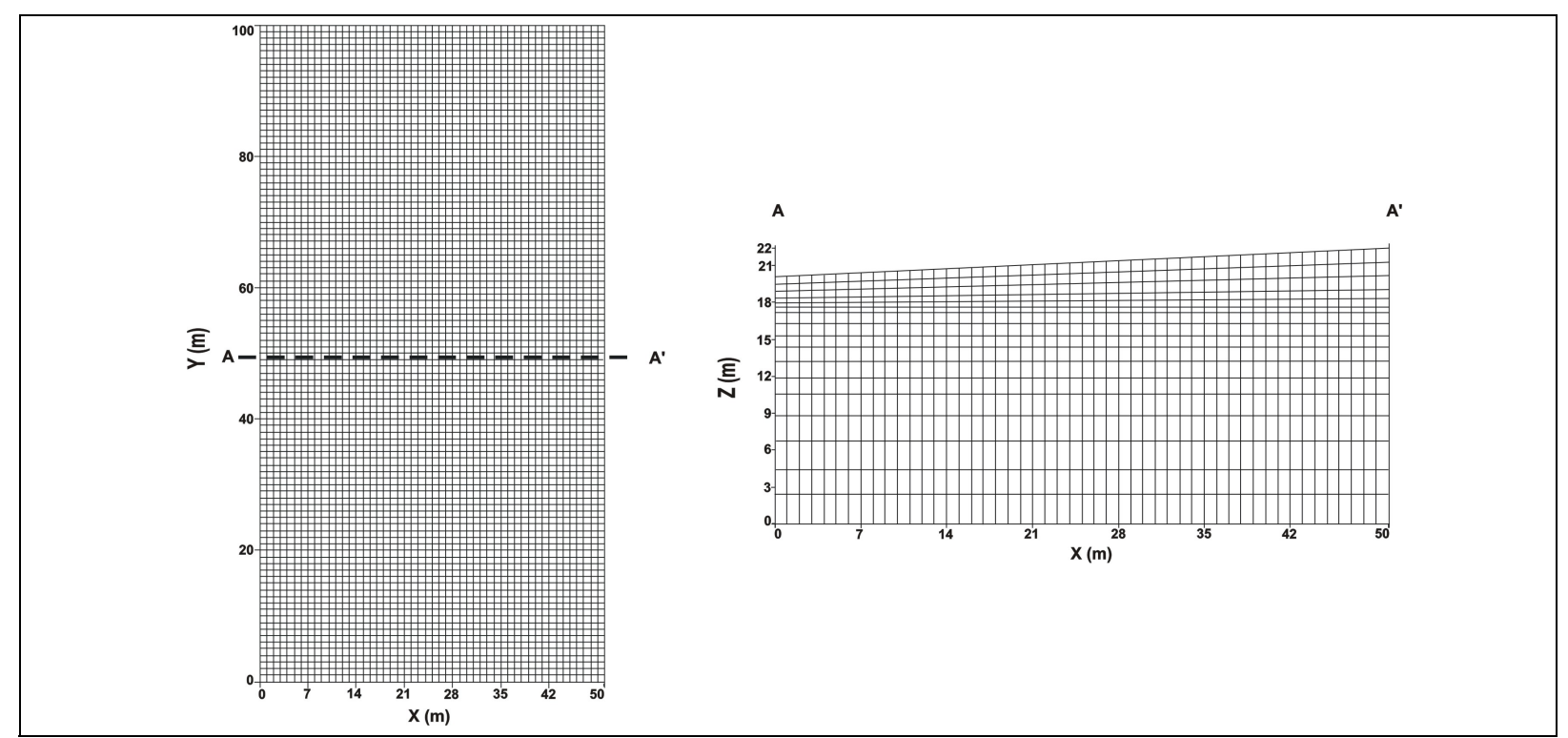

Figura 2- Características geométricas da porção $P$ do modelo M4 (planta e perfil A-A')

Figure 2-Geometry of portion P of the M4 model (plan view and vertical profile A-A') 


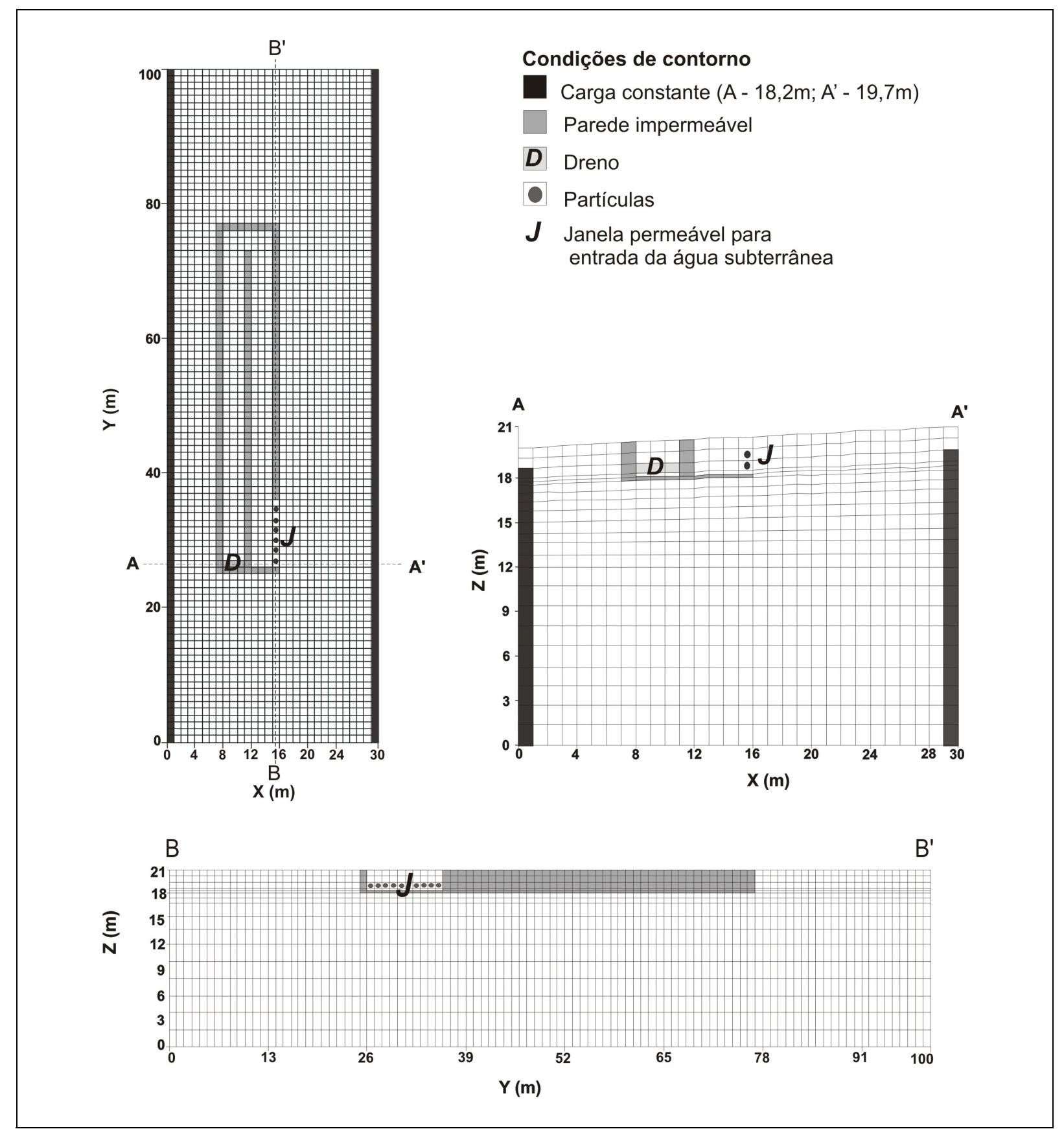

Figura 3- Geometria e características hidráulicas do modelo M1 (planta, perfil A-A' e perfil B-B', respectivamente)

Figure 3- Geometry and hydraulic characteristics of the MI model (plan view and vertical profile A-A')

Nos modelos M2 e M3, que são uma variação de M1, construiu-se uma barreira em forma de cone (Figuras 4 e 5) para aumentar horizontalmente a abertura $J$. 


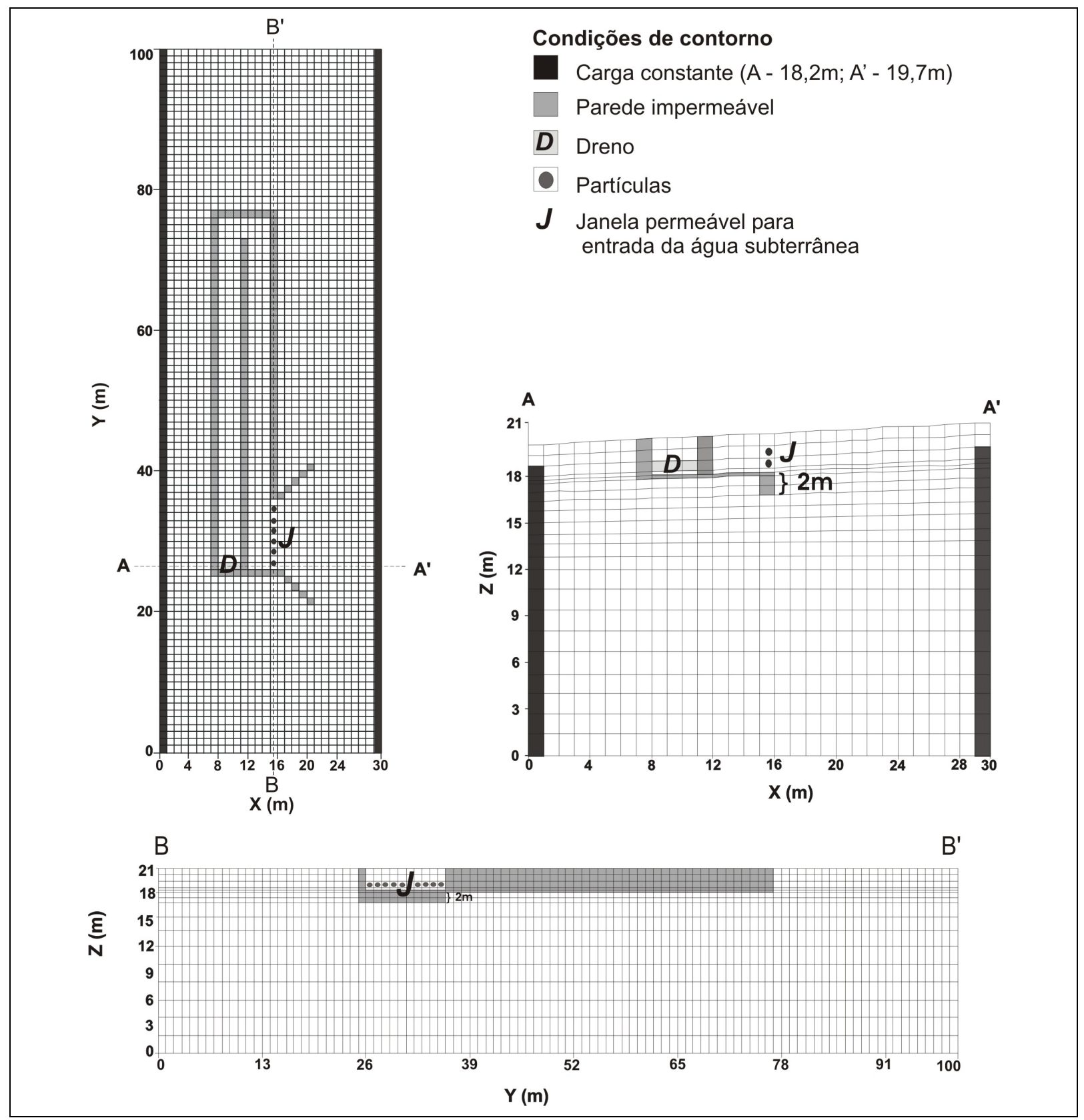

Figura 4- Geometria e características hidráulicas do modelo M2 (planta, perfil A-A' e perfil B-B', respectivamente)

Figure 4- Geometry and hydraulic characteristics of the M2 model (plan view and vertical profile A-A' and B-B', respectively) 

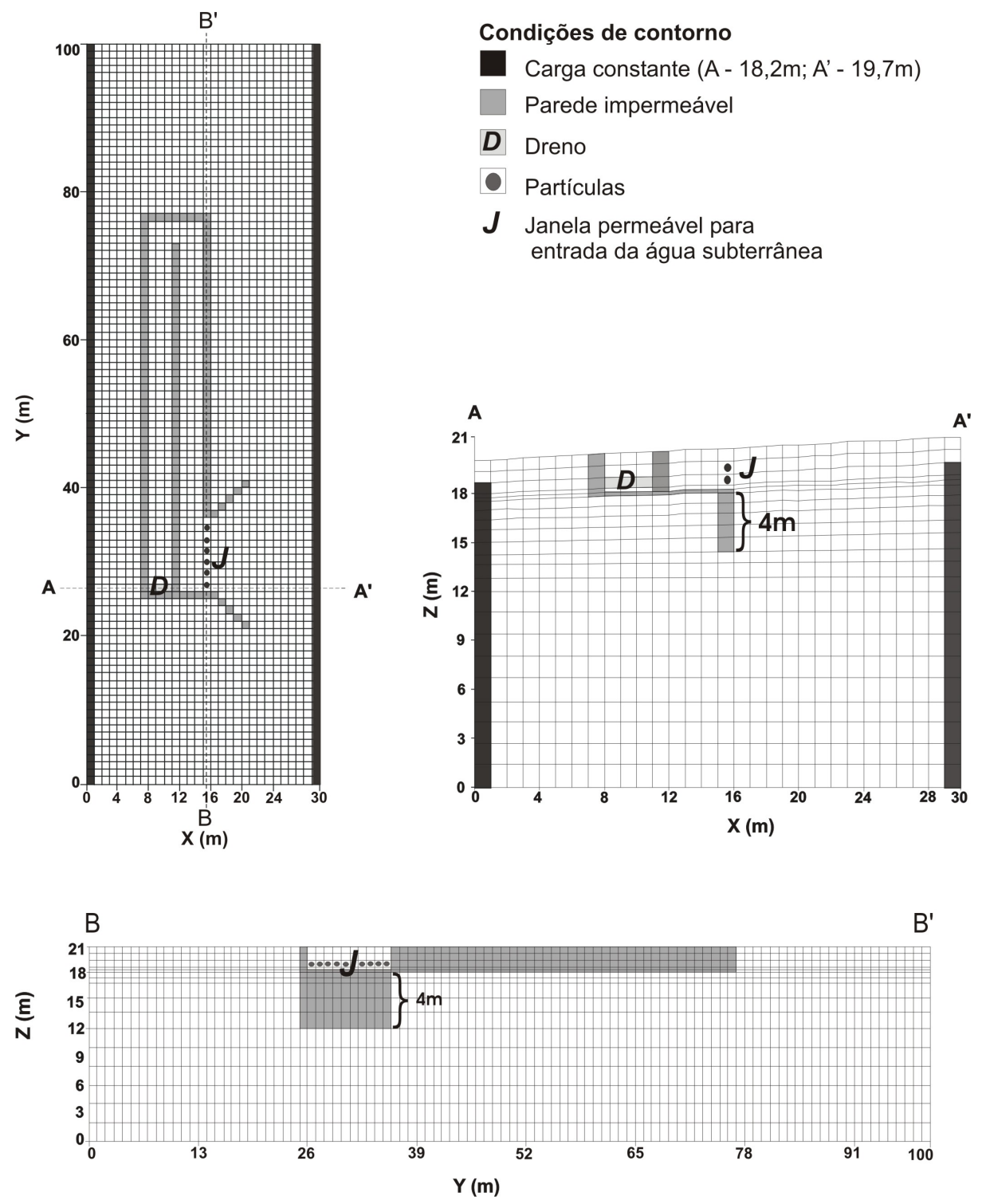

Figura 5- Geometria e características hidráulicas do modelo M3 (planta, perfil A-A' e perfil B-B', respectivamente)

Figure 5- Geometry and hydraulic characteristics of the M3 model (plan view and vertical profile A-A' and B-B', respectively)

Uma barreira impermeável também foi inserida imediatamente abaixo da janela $J$, estendendo-se até $2 \mathrm{~m}$ (M2) e $4 \mathrm{~m}$ (M3) de profundidade, além do fundo do canal escavado. O objetivo dessa inclusão foi testar a influência dessas barreiras no aumento da eficiência da cavidade em capturar água subterrânea.

Como os resultados dessa comparação não foram satisfatórios para a relação volume de água tratada ( $\geq 10 \mathrm{~m}^{3} /$ dia) e tempo de residência (entre 10 e 15 dias, aproximadamente), criou-se o modelo M4 (Figura 6). Este modelo simulou um sistema integrado de wetlands, com um sistema de fluxo superficial seguido de células de fluxo subsuperficial com solo filtrante, considerado mais efetivo no tratamento de águas contaminadas por mais de um composto (TCHOBANOGLOUS, 1993).

As dimensões de M4 foram resultados da busca por $Q_{\text {dreno }} \geq 10 \mathrm{~m}^{3} /$ dia e $t_{\text {trânsito }}$ entre 10 e 15 dias. A geometria do sistema foi baseada em um sistema existente, mas inoperante. A altura do dreno foi escolhida de modo a manter uma coluna de água de 0,90 m no interior do canal de fluxo superficial. Não se utilizou recarga neste modelo.

As características hidráulicas e geométricas dos quatro modelos, em regime estacionário de cargas, estão sumarizadas na tabela 2. 


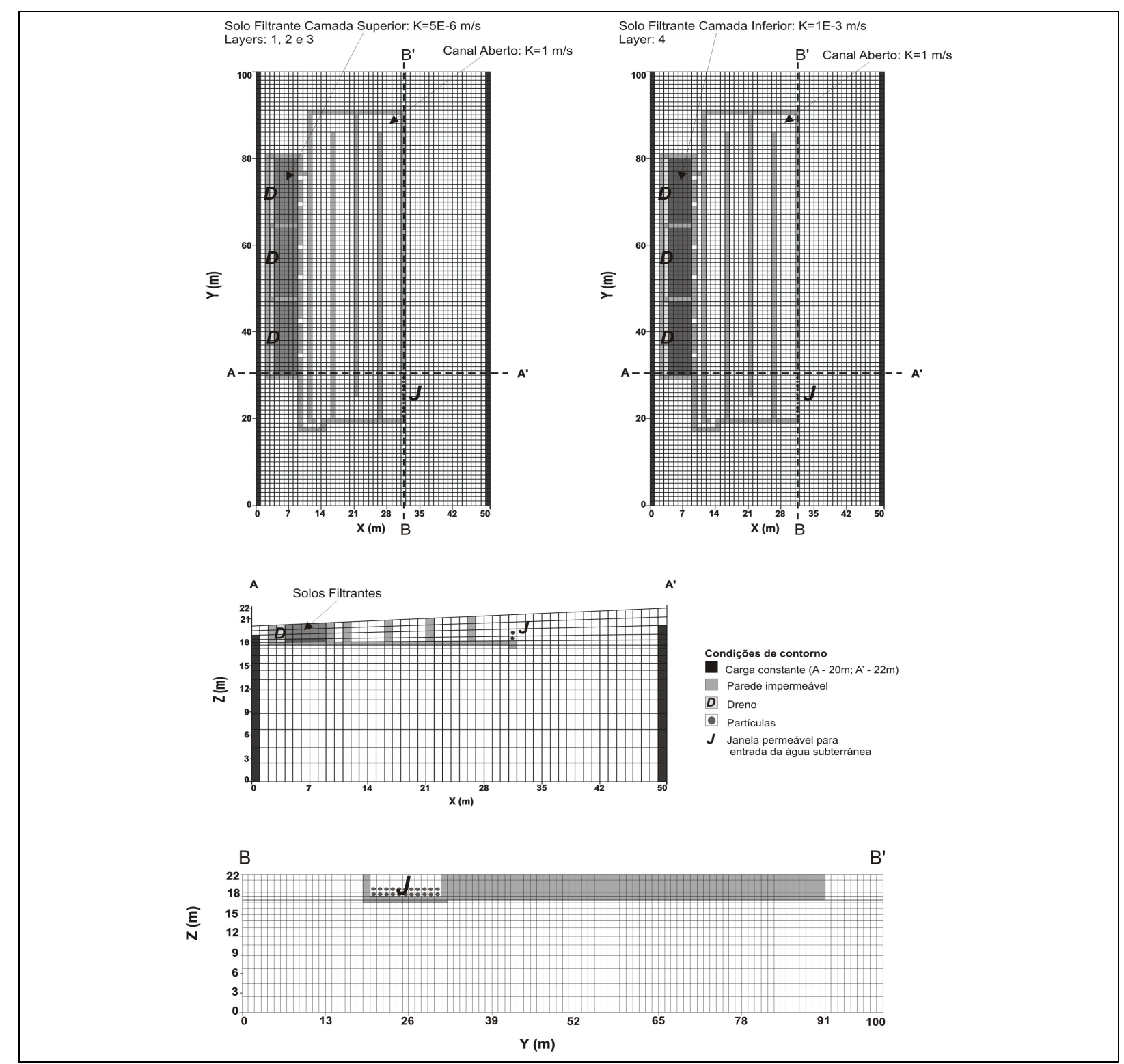

Figura 6- Geometria e características hidráulicas do modelo M4 (planta, perfil A-A' e perfil B-B', respectivamente)

Figure 6- Geometry and hydraulic characteristics of the M4 model (plan view and vertical profile A-A' and B-B', respectively

Tabela 2- Características geométricas e hidráulicas dos modelos criados

Table 2- Geometry and hydraulic characteristics for each simulation

\begin{tabular}{c|c|c|c|c}
\hline Modelo & $\begin{array}{c}\text { Tipo de } \\
\text { wetland } \\
\text { simulada }\end{array}$ & \multicolumn{1}{|c|}{ Forma geométrica } & $\begin{array}{c}\text { Dimensões } \\
(\mathbf{m})\end{array}$ & $\begin{array}{c}\text { K canal } \\
\text { escavado (m/s) }\end{array}$ \\
\hline M1 & SFS & Semelhante a um $\cap$ & $100 \times 3$ & 1 \\
\hline M2 & SFS & $\begin{array}{l}\text { Semelhante a um } \cap \text { com barreira horizontal em forma de } \\
\text { cone na janela }(J) \text { e barreira vertical de 2 m de } \\
\text { profundidade logo abaixo de } J\end{array}$ & $100 \times 3$ & 1 \\
\hline M3 & SFS & $\begin{array}{l}\text { Semelhante a um } \cap \text {, com barreira horizontal em forma de } \\
\text { cone na janela }(J) \text { e barreira vertical de 4 m de } \\
\text { profundidade logo abaixo de } J \text {. }\end{array}$ & $100 \times 3$ & 1 \\
\hline \multirow{2}{*}{ M4 } & SFS + & $\begin{array}{l}\text { FS - semelhante a um M, com barreira vertical de 1 m de } \\
\text { profundidade logo abaixo da janela } J .\end{array}$ & $280 \times 4$ & 1 \\
\cline { 3 - 5 } & SFSS & Retangulares, dispostos em paralelo & $17 \times 6$ & $1 \times 10^{-3}$ (base) \\
\cline { 3 - 5 }
\end{tabular}

SFS - Sistema de fluxo superficial

SFSS - sistema de fluxo subsuperficial

$J$ - abertura permeável de $10 \mathrm{~m}$ de largura, para permitir a entrada da água subterrânea na wetland. 
Para verificar as condições nas quais a wetland seria uma alternativa viável, ou não, para cada um dos modelos foram empregadas combinações distintas dos parâmetros $\mathrm{K}_{\text {terreno }} \mathrm{e}$ $\mathrm{H}_{\text {dreno. }}$ Os valores de condutividade hidráulica do terreno utilizados variaram de $1 \times 10^{-6} \mathrm{~m} / \mathrm{s}$ (material siltoso) a $1 \times 10^{3} \mathrm{~m} / \mathrm{s}$ (material arenoso) (FREEZE; CHERRY, 1979).

\section{Confiabilidade dos Resultados}

Como os modelos simulados não foram calibrados, por serem hipotéticos, observou-se a porcentagem de discrepância entre o volume de água que entrava $\left(\mathrm{Q}_{\text {entrada }}\right)$ e o que saía $\left(\mathrm{Q}_{\text {sáda }}\right)$ da porção $R$ do aquiífero. Os resultados foram todos inferiores a $0,51 \%$, indicando a representatividade dos resultados.

$\mathrm{O}$ volume de água que entrou em $R$ não se alterou nas situações sem recarga e com recarga. Já o volume de água drenado foi de 5 a $9 \%$ maior para as simulações com recarga, $(250 \mathrm{~mm} / \mathrm{ano})$ para condutividade de $1 \times 10^{-6} \mathrm{~m} / \mathrm{s}$.

As variações de $Q_{\text {dreno }} e Q_{\text {entrada }}$ foram praticamente idênticas para os modelos M1, M2 e $\mathrm{M} 3$, e só não foram lineares nos casos de $\mathrm{H}_{\text {dreno }}=$ $1 \mathrm{~m}$ e na situação de recarga para condutividade hidráulica $\left(\mathrm{K}_{\text {terreno }}\right)$ de $1 \times 10^{-6} \mathrm{~m} / \mathrm{s}$. A porcentagem de $\mathrm{Q}_{\text {dreno }} / \mathrm{Q}_{\text {entrada }}$ permaneceu na ordem de 1 a $2 \%$ para meios de alto $K_{\text {terreno }}\left(1 \times 10^{-3} \mathrm{~m} / \mathrm{s}\right)$, quer se utilizasse ou não de recarga. Nos casos intermediários de $\mathrm{K}_{\text {terreno }}\left(1 \times 10^{-5}\right.$ e $\left.1 \times 10^{-4} \mathrm{~m} / \mathrm{s}\right)$, a diferença entre as simulações sem recarga e com recarga não chegou a $1 \%$ em cada um dos três modelos.

\section{Resultados das Simulações Matemáticas}

Para os modelos M1, M2 e M3, as simulações foram divididas em dois grupos: sem recarga e com recarga $(250 \mathrm{~mm} / \mathrm{ano})$. Largura, profundidade da pluma e tempo de residência foram medidos através do caminhamento de partículas colocadas na janela permeável $(J)$ do canal e processadas pelo módulo Modpath.

Devido à posição das partículas, suas trajetórias foram simuladas para frente (forward) e para trás (backward), cujos resultados estão representados nas Figuras 7 a 9. O caminhamento "para frente" foi utilizado para medir os tempos mínimo e máximo de permanência da água dentro da wetland. O caminhamento para trás serviu para auxiliar na medição da profundidade e largura de captura de uma possível pluma contaminante.

$\mathrm{O}$ volume de água tratado, obtido através do balanço de massas realizado pelo módulo ZBUD, correspondeu à quantidade de água que passou pelos drenos posicionados nas saídas dos canais.

As alturas dos drenos variaram de modo a manter colunas de água de $1 \mathrm{~m}, 0,75 \mathrm{~m}, 0,5 \mathrm{~m}$ e $0,3 \mathrm{~m}$ dentro do canal dos modelos M1, M2 e M3.

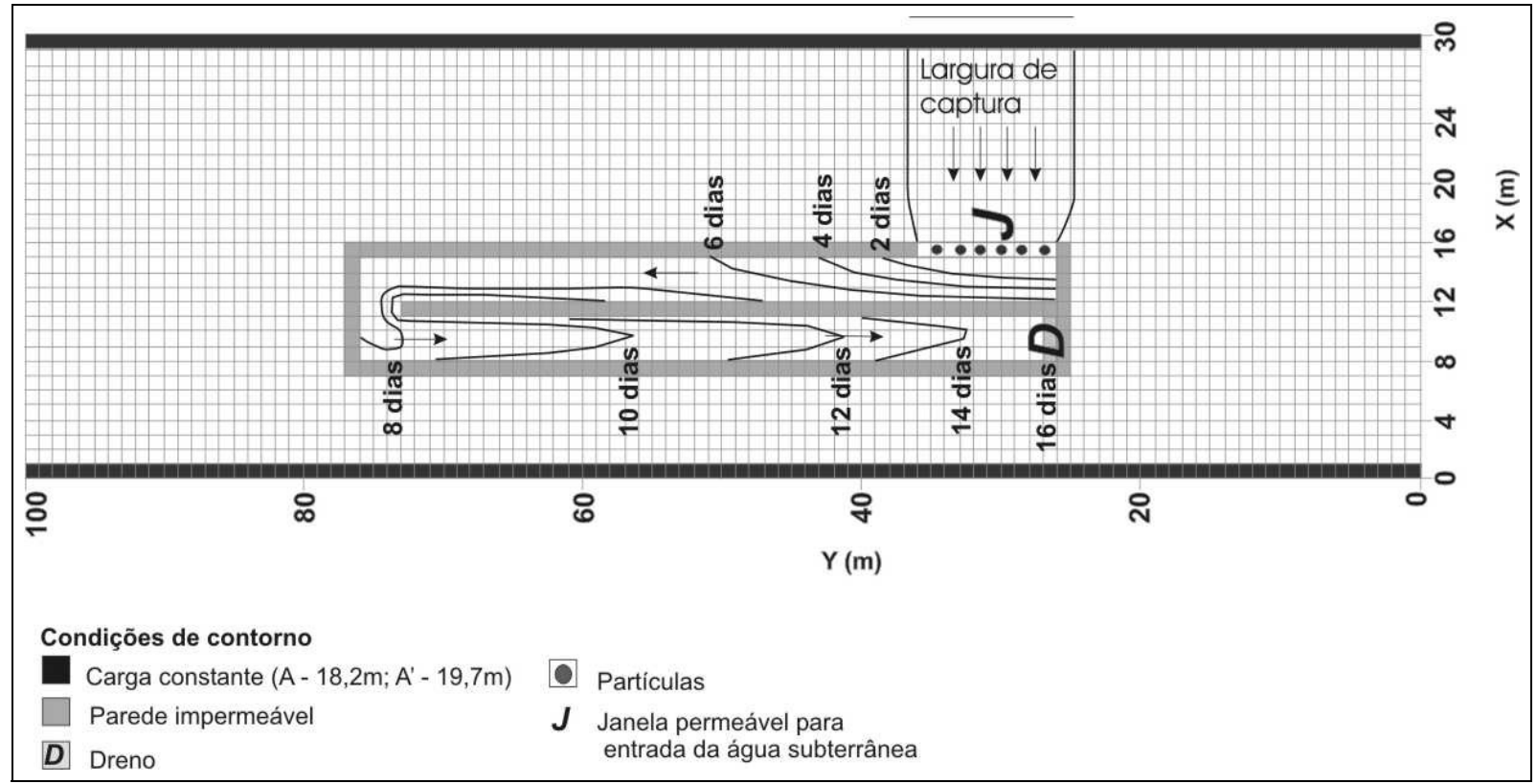

Figura 7- Exemplo de resultado das trajetórias das partículas para o modelo M1

Figure 7- Example of results of the particle tracking trajectory for the M1 model 


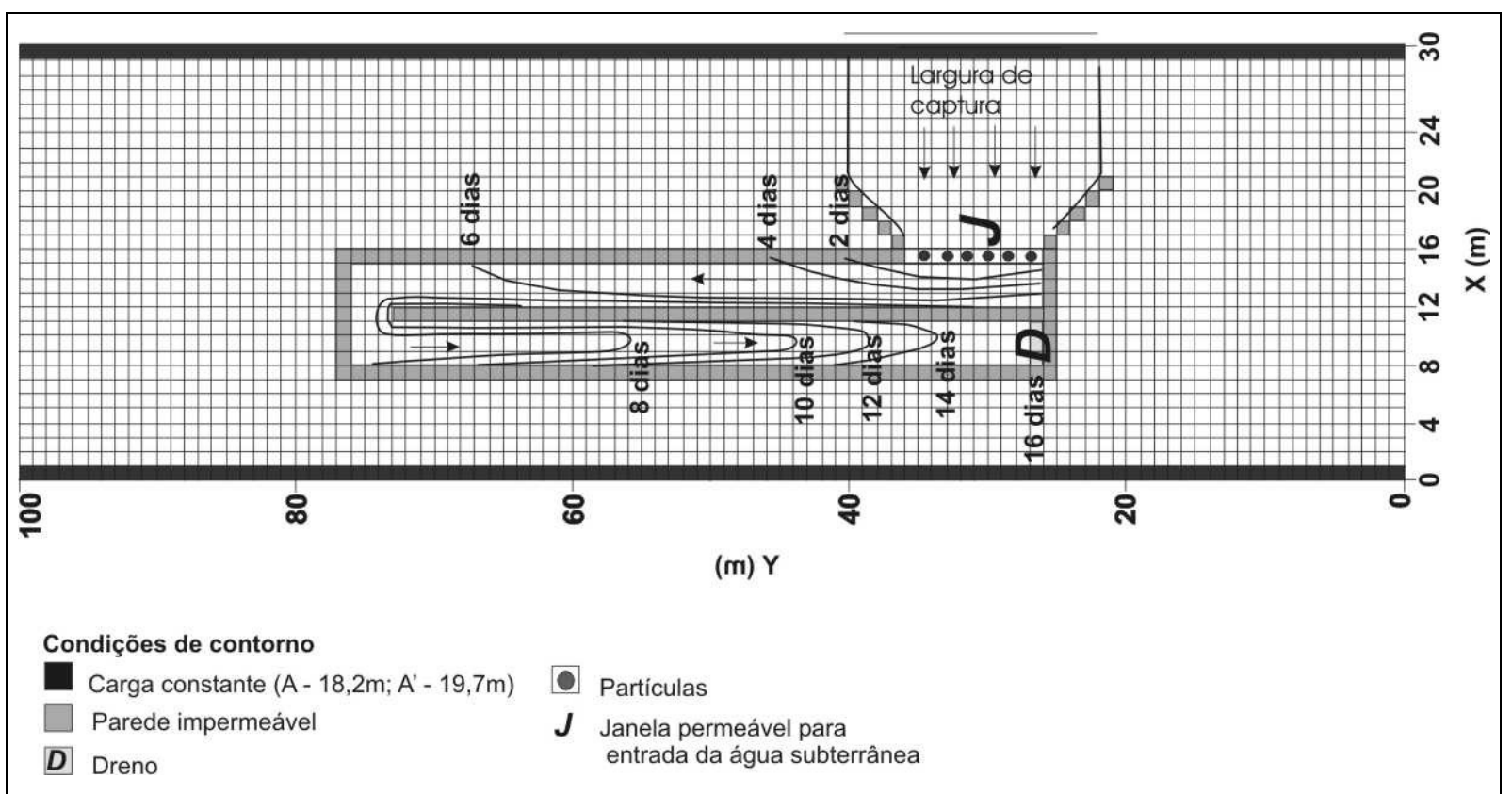

Figura 8- Exemplo de resultado das trajetórias das partículas para o modelo M2

Figure 8- Example of results of the particle tracking trajectory for the M2 model

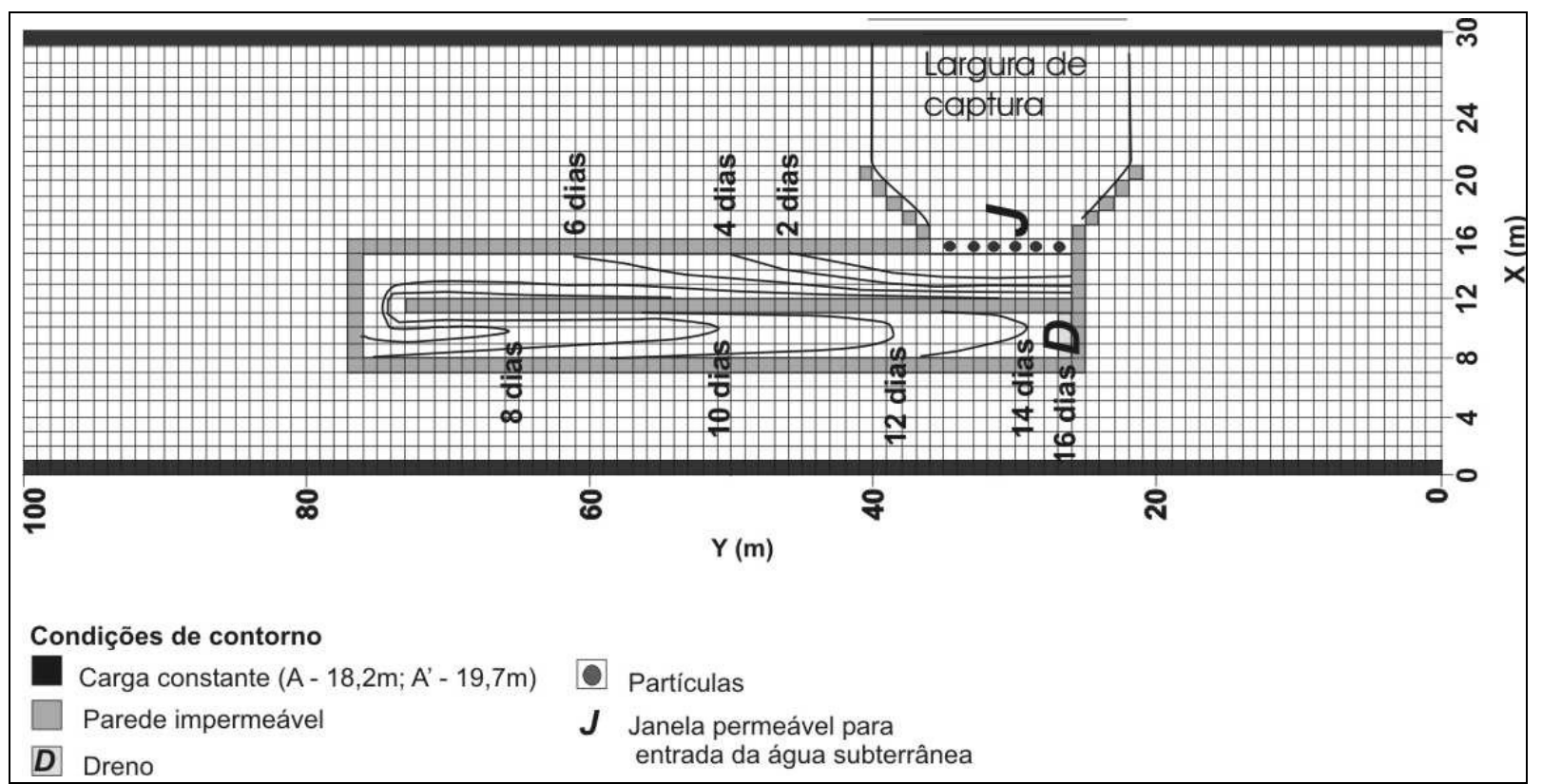

Figura 9- Exemplo de resultado das trajetórias das partículas para o modelo M3

Figure 9-Example of results of the particle tracking trajectory for the M3 model

\section{Volume de Água Tratada x Tempo de Residência}

Na comparação entre M1, M2 e M3, a vazão de água drenada $\left(\mathrm{Q}_{\text {dreno }}\right)$ e o tempo de trânsito mínimo e máximo das partículas dentro do canal $\left(t_{\text {trânsito }}\right)$ foram inversamente proporcionais entre si e controlados pela condutividade hidráulica da região $\left(\mathrm{K}_{\text {terreno }}\right)$. Os tempos mínimo e máximo determinados corresponderam àqueles em que a primeira e a última partícula atingiram o dreno, respectivamente.

Estas relações foram também avaliadas de acordo com as quatro alturas do dreno $\left(\mathrm{H}_{\text {dreno }}\right)$ dentro do canal. As curvas dos resultados $\mathrm{Q}_{\text {dreno }} \mathrm{e}$

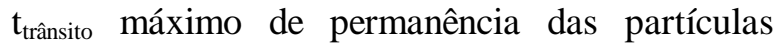
dentro do canal como funções de $\mathrm{K}_{\text {terreno }}$ (para diferentes $\mathrm{H}_{\text {dreno }}$ ) ilustram como este fator governa aqueles dois parâmetros, conforme ilustrado na Figura 10.

$\mathrm{H}_{\text {dreno }}$ também influenciou $\mathrm{Q}_{\text {dreno }}$ e $\mathrm{t}_{\text {trânsito: }}$ : quanto menor a coluna de água dentro da wetland, menor o tempo de trânsito e maior o volume de água que passa pelo dreno, devido ao maior gradiente hidráulico. 


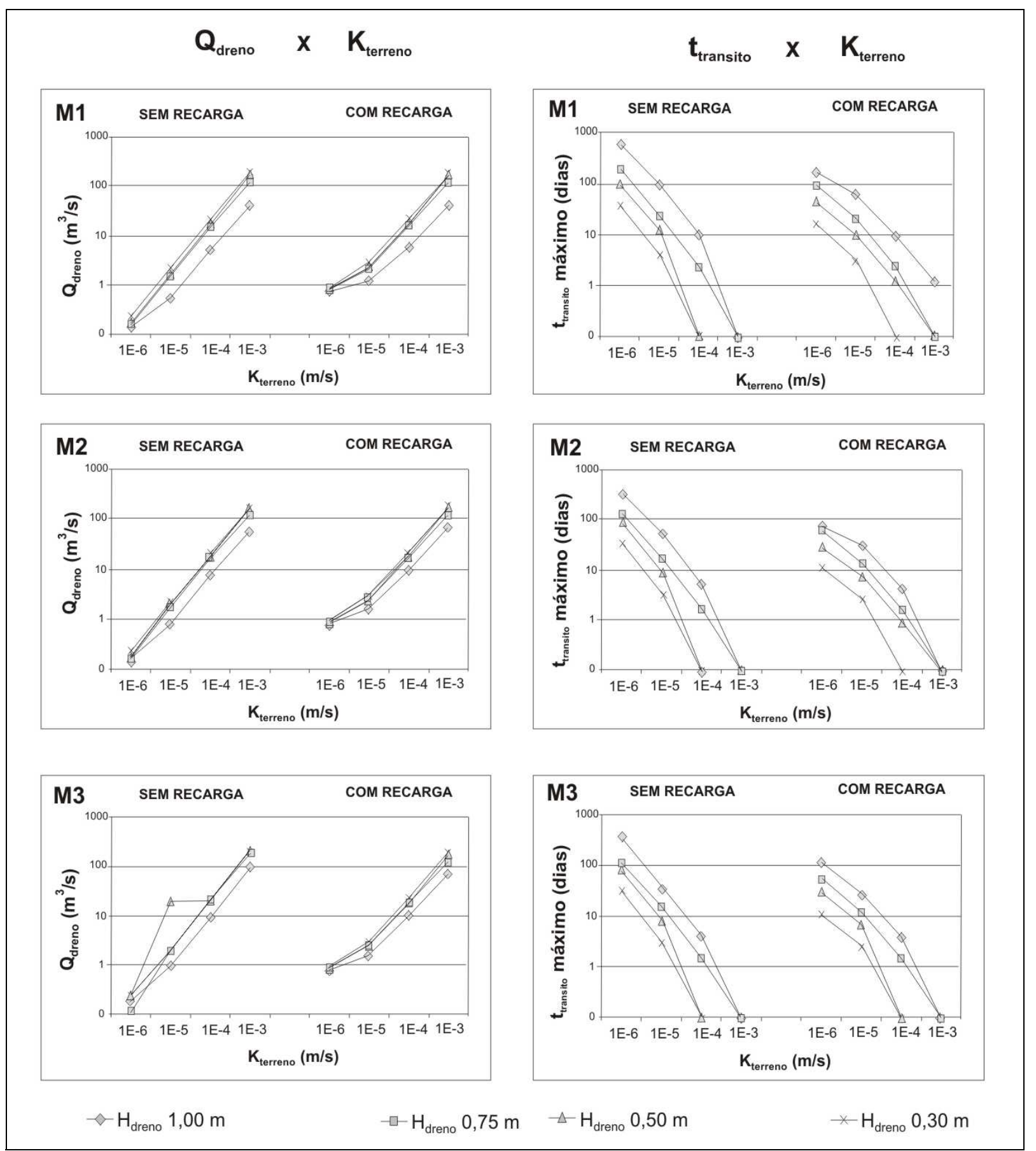

Figura 10- Volume da água drenada para os modelos e Tempo de trânsito para os modelos M1, M2 e M3 em função da condutividade hidráulica para diferentes alturas de drenos

Figure 10-Volume of drained water and transit time through the wetland for the M1, M2 and M3 models in function of the hydraulic conductivity and different drain heigh

Neste trabalho considerou-se como ideal $\mathrm{t}_{\text {trânsito }}$ entre 10 e 15 dias, aproximadamente. Os resultados que mais se aproximaram desse intervalo foram os do modelo M1 com $\mathrm{K}_{\text {terreno }}=1 \times 10^{-5} \mathrm{~m} / \mathrm{s}, \quad \mathrm{H}_{\text {dreno }}=0,50 \mathrm{~m}$, sem recarga, cujo intervalo de tempo das partículas esteve entre 8,94 e 13 dias e $K_{\text {terreno }}=1 \times 10^{-6} \mathrm{~m} / \mathrm{s}$, $\mathrm{H}_{\text {dreno }}=0,30 \mathrm{~m}$, com recarga, com intervalo de tempo entre 11 e 16,3 dias, e também do modelo M3 com $\mathrm{K}_{\text {terreno }}=1 \times 10^{-5} \mathrm{~m} / \mathrm{s}, \mathrm{H}_{\text {dreno }}=0,75 \mathrm{~m}$, com recarga, com intervalo de tempo entre 11,9 e 16,5 dias (Tabela 3). 
Tabela 3- Variação no tempo de trânsito das partículas (tempo mínimo e máximo) dentro do canal escavado para os modelos M1, M2 e M3

Table 3-Variation of transit time of particle tracking (minimum and maximum time) in the interior of channel for the M1, M2 e M3 models

\begin{tabular}{|c|c|c|c|c|c|c|c|}
\hline \multirow{4}{*}{$\begin{array}{c}\mathbf{H}_{\text {dreno }} \\
(\mathbf{m})\end{array}$} & \multirow{4}{*}{$\begin{array}{c}\mathbf{K}_{\text {terreno }} \\
(\mathbf{m} / \mathbf{s})\end{array}$} & \multicolumn{6}{|c|}{ Modelo } \\
\hline & & \multicolumn{2}{|c|}{ M1 } & \multicolumn{2}{|c|}{ M2 } & \multicolumn{2}{|c|}{ M3 } \\
\hline & & \multicolumn{2}{|c|}{$\mathbf{t}_{\text {dreno }}(\operatorname{dias})$} & \multicolumn{2}{|c|}{$\mathbf{t}_{\text {dreno }}($ dias $)$} & \multicolumn{2}{|c|}{$t_{\text {dreno }}(\operatorname{dias})$} \\
\hline & & $\begin{array}{c}\text { Sem } \\
\text { recarga }\end{array}$ & $\begin{array}{c}\text { Com } \\
\text { recarga }\end{array}$ & $\begin{array}{c}\text { Sem } \\
\text { recarga }\end{array}$ & $\begin{array}{c}\text { Com } \\
\text { recarga }\end{array}$ & $\begin{array}{c}\text { Sem } \\
\text { recarga }\end{array}$ & $\begin{array}{c}\text { Com } \\
\text { recarga }\end{array}$ \\
\hline \multirow{4}{*}{1,00} & $1 \times 10^{-6}$ & 421 a 600 & 122 a 170 & 317 a 430 & 72,0 a 120 & 351 a 452 & 112 a 170 \\
\hline & $1 \times 10^{-5}$ & 74,7 a 97,0 & 42,6 a 63,0 & 50,2 a 65,5 & 29,8 a 35,0 & 36,2 a 48,5 & 28,1 a 48,0 \\
\hline & $1 \times 10^{-4}$ & 6,90 a 10,5 & 6,44 a 9,50 & 5,07 a 7,50 & 4,21 a 4,90 & 4,00 a 5,00 & 3,82 a 5,80 \\
\hline & $1 \times 10^{-3}$ & $<1,00$ & 0,91 a 1,20 & $<1,00$ & $<1,00$ & $<1,00$ & $<1,00$ \\
\hline \multirow{4}{*}{0,75} & $1 \times 10^{-6}$ & 140 a 205 & 57,7 a 94,0 & 126 a 160 & 58,0 a 92,0 & 115 a 172 & 51,5 a 84,0 \\
\hline & $1 \times 10^{-5}$ & 17,2 a 23,0 & 13,7 a 21,0 & 15,9 a 20,0 & 13,0 a 19,7 & 14,9 a 19,0 & 11,9 a 16,5 \\
\hline & $1 \times 10^{-4}$ & 1,73 a 2,40 & 1,69 a 2,50 & 1,65 a 2,30 & 1,62 a 2,40 & 1,50 a 2,20 & 1,50 a 2,10 \\
\hline & $1 \times 10^{-3}$ & $<1,00$ & $<1,00$ & $<1,00$ & $<1,00$ & $<1,00$ & $<1,00$ \\
\hline \multirow{4}{*}{0,50} & $1 \times 10^{-6}$ & 79,0 a 100 & 29,5 a 46,5 & 82,5 a 100 & 28,0 a 41,0 & 80,3 a 104 & 30,2 a 53,0 \\
\hline & $1 \times 10^{-5}$ & 8,94 a 13,0 & 7,26 a 10,0 & 8,53 a 11,0 & 7,27 a 9,60 & 8,30 a 11,0 & 6,59 a 9,20 \\
\hline & $1 \times 10^{-4}$ & $<1,00$ & 0,91 a 1,30 & $<1,00$ & 0,90 a 1,30 & $<1,00$ & $<1,00$ \\
\hline & $1 \times 10^{-3}$ & $<1,00$ & $<1,00$ & $<1,00$ & $<1,00$ & $<1,00$ & $<1,00$ \\
\hline \multirow{4}{*}{0,30} & $1 \times 10^{-6}$ & 29,7 a 37,0 & 11,0 a 16,3 & 32,3 a 40,0 & 11,1 a 15,5 & 32,2 a 46,0 & 11,0 a 16,3 \\
\hline & $1 \times 10^{-5}$ & 3,14 a 4,00 & 2,61 a 3,30 & 3,15 a 4,00 & 2,46 a 3,5 & 3,08 a 4,30 & 2,57 a 3,50 \\
\hline & $1 \times 10^{-4}$ & $<1,00$ & $<1,00$ & $<1,00$ & $<1,00$ & $<1,00$ & $<1,00$ \\
\hline & $1 \times 10^{-3}$ & $<1,00$ & $<1,00$ & $<1,00$ & $<1,00$ & $<1,00$ & $<1,00$ \\
\hline
\end{tabular}

$\mathrm{H}_{\text {dreno }}=$ coluna de água no interior do canal

$\mathrm{K}_{\text {terreno }}=$ condutividade hidráulica do terreno

$\mathrm{T}_{\text {trânsito }}=$ tempo de trânsito (tempo mínimo a tempo máximo)

Quanto às vazões dos cenários com melhores resultados de $t_{\text {trânsito, }} o$ melhor resultado foi $o$ do

modelo $\mathrm{M} 3$, com $\mathrm{Q}_{\text {dreno }}$ de $2,44 \mathrm{~m}^{3} /$ dia, como mostra a Tabela 4.

Tabela 4- Variação na vazão drenada para M1, M2 e M3

Table 4- Flowrate variation for M1, M2 and M3 models

\begin{tabular}{|c|c|c|c|c|c|c|c|}
\hline \multirow{4}{*}{$\mathbf{H}_{\text {dreno }}(\mathbf{m})$} & \multirow{4}{*}{$\begin{array}{c}\mathbf{K}_{\text {terreno }} \\
(\mathbf{m} / \mathbf{s})\end{array}$} & \multicolumn{6}{|c|}{ Modelo } \\
\hline & & \multicolumn{2}{|c|}{ M1 } & \multicolumn{2}{|c|}{ M2 } & \multicolumn{2}{|c|}{ M3 } \\
\hline & & \multicolumn{2}{|c|}{$\mathbf{Q}_{\text {dreno }}\left(\mathrm{m}^{3} / \mathrm{dia}\right)$} & \multicolumn{2}{|c|}{$Q_{\text {dreno }}\left(\mathbf{m}^{3} /\right.$ dia $)$} & \multicolumn{2}{|c|}{$Q_{\text {dreno }}\left(\mathrm{m}^{3} / \mathrm{dia}\right)$} \\
\hline & & $\begin{array}{c}\text { Sem } \\
\text { recarga }\end{array}$ & $\begin{array}{c}\text { Com } \\
\text { recarga }\end{array}$ & $\begin{array}{c}\text { Sem } \\
\text { recarga }\end{array}$ & $\begin{array}{c}\text { Com } \\
\text { recarga }\end{array}$ & $\begin{array}{c}\text { Sem } \\
\text { recarga }\end{array}$ & $\begin{array}{c}\text { Com } \\
\text { recarga }\end{array}$ \\
\hline \multirow{4}{*}{1,00} & $1 \times 10^{-6}$ & 0,14 & 0,73 & 0,16 & 0,76 & 0,18 & 0,76 \\
\hline & $1 \times 10^{-5}$ & 0,54 & 1,21 & 0,74 & 1,53 & 0,92 & 1,60 \\
\hline & $1 \times 10^{-4}$ & 5,25 & 5,90 & 7,22 & 9,09 & 9,10 & 9,81 \\
\hline & $1 \times 10^{-3}$ & 43,0 & 41,0 & 57,7 & 71,7 & 98,7 & 74,2 \\
\hline \multirow[t]{4}{*}{0,75} & $1 \times 10^{-6}$ & 0,15 & 8,82 & 0,15 & 0,82 & 0,11 & 8,85 \\
\hline & $1 \times 10^{-5}$ & 1,48 & 2,15 & 1,62 & 2,29 & 1,93 & 2,44 \\
\hline & $1 \times 10^{-4}$ & 15,5 & 15,7 & 15,8 & 16,4 & 20,5 & 17,8 \\
\hline & $1 \times 10^{-3}$ & 117 & 118 & 126 & 127 & 178 & 135 \\
\hline \multirow{4}{*}{0,50} & $1 \times 10^{-6}$ & 0,17 & 0,83 & 0,17 & 0,84 & 0,23 & 0,84 \\
\hline & $1 \times 10^{-5}$ & 1,68 & 2,35 & 1,78 & 2,36 & 1,93 & 2,46 \\
\hline & $1 \times 10^{-4}$ & 16,9 & 17,5 & 17,2 & 17,8 & 19,5 & 18,9 \\
\hline & $1 \times 10^{-3}$ & 177 & 178 & 179 & 180 & 216 & 193 \\
\hline \multirow{4}{*}{0,30} & $1 \times 10^{-6}$ & 0,23 & 0,88 & 0,21 & 0,88 & 0,23 & 0,90 \\
\hline & $1 \times 10^{-5}$ & 2,18 & 2,87 & 2,19 & 2,86 & 1,93 & 2,93 \\
\hline & $1 \times 10^{-4}$ & 21,9 & 21.7 & 20,7 & 21,4 & 19,5 & 22,2 \\
\hline & $1 \times 10^{-3}$ & 190 & 191 & 176 & 180 & 216 & 204 \\
\hline
\end{tabular}

$\mathrm{H}_{\text {dreno }}=$ coluna de água no interior do canal

$\mathrm{K}_{\text {terreno }}=$ condutividade hidráulica do terreno

$\mathrm{T}_{\text {trânsito }}=$ tempo de trânsito 


\section{Dimensões das Plumas Capturadas}

As profundidades de captura das partículas ( $\left.\mathrm{P}_{\text {captura }}\right)$ nos modelos M1, M2 e M3 variaram de 0,7 a 4,0 $\mathrm{m}$ nas simulações sem recarga e de 0,2 a 3,6 $\mathrm{m}$ com recarga (Tabela 5).

Os resultados mostraram que $\mathrm{P}_{\text {captura }}$ foi maior para $H_{\text {dreno }}=0,3 \mathrm{~m}$ e menor quando $H_{\text {dreno }}=1 \mathrm{~m}$ para os três modelos. A diferença nas profundidades foi de $1,5 \mathrm{~m}$ considerando que houve recarga, para o modelo M3, que utilizou uma barreira impermeável de $4 \mathrm{~m}$ abaixo da janela $\mathrm{J}$ da wetland, e 2,9 $\mathrm{m}$ quando a recarga foi nula, para M1, que não continha barreira.

Tabela 5- Variação na profundidade de captura das partículas para M1, M2 e M3

Table 5- Depth variation of capture zone for M1, M2 e M3 models

\begin{tabular}{|c|c|c|c|c|c|c|c|}
\hline \multirow{4}{*}{$\mathbf{H}_{\text {dreno }}(\mathbf{m})$} & \multirow{4}{*}{$\begin{array}{c}\mathbf{K}_{\text {terreno }} \\
(\mathbf{m} / \mathbf{s})\end{array}$} & \multicolumn{6}{|c|}{ Modelo } \\
\hline & & \multicolumn{2}{|c|}{ M1 } & \multicolumn{2}{|c|}{ M2 } & \multicolumn{2}{|c|}{ M3 } \\
\hline & & \multicolumn{2}{|c|}{$\mathbf{P}_{\text {captura }}(\mathbf{m})$} & \multicolumn{2}{|c|}{$\mathbf{P}_{\text {captura }}(\mathbf{m})$} & \multicolumn{2}{|c|}{$\mathbf{P}_{\text {captura }}(\mathbf{m})$} \\
\hline & & $\begin{array}{c}\text { Sem } \\
\text { recarga }\end{array}$ & $\begin{array}{c}\text { Com } \\
\text { recarga }\end{array}$ & $\begin{array}{c}\text { Sem } \\
\text { recarga }\end{array}$ & $\begin{array}{c}\text { Com } \\
\text { recarga }\end{array}$ & $\begin{array}{c}\text { Sem } \\
\text { recarga }\end{array}$ & $\begin{array}{c}\text { Com } \\
\text { recarga }\end{array}$ \\
\hline \multirow{4}{*}{1,00} & $1 \times 10^{-6}$ & 0,70 & 0,20 & 1,50 & 0,30 & 2,00 & 0,70 \\
\hline & $1 \times 10^{-5}$ & 1,20 & 1,00 & 1,50 & 1,40 & 2,00 & 1,90 \\
\hline & $1 \times 10^{-4}$ & 1,20 & 1,10 & 1,50 & 1,50 & 2,00 & 2,00 \\
\hline & $1 \times 10^{-3}$ & 0,70 & 0,90 & 1,70 & 1,30 & 2,00 & 1,70 \\
\hline \multirow{4}{*}{0,75} & $1 \times 10^{-6}$ & 2,70 & 1,80 & 2,80 & 2,90 & 3,70 & 2,30 \\
\hline & $1 \times 10^{-5}$ & 2,80 & 2,70 & 2,80 & 2,80 & 3,80 & 3,20 \\
\hline & $1 \times 10^{-4}$ & 2,70 & 2,70 & 2,80 & 2,80 & 3,70 & 3,20 \\
\hline & $1 \times 10^{-3}$ & 2,20 & 2,20 & 2,30 & 2,30 & 3,70 & 2,70 \\
\hline \multirow{4}{*}{0,50} & $1 \times 10^{-6}$ & 3,00 & 1,80 & 3,00 & 1,70 & 3,30 & 2,20 \\
\hline & $1 \times 10^{-5}$ & 2,90 & 2,90 & 2,90 & 2,80 & 3,30 & 3,20 \\
\hline & $1 \times 10^{-4}$ & 2,90 & 2,90 & 2,90 & 2,90 & 3,30 & 3,20 \\
\hline & $1 \times 10^{-3}$ & 3,10 & 3,10 & 3,10 & 3,10 & 3,30 & 3,50 \\
\hline \multirow{4}{*}{0,30} & $1 \times 10^{-6}$ & 3,60 & 2,50 & 3,60 & 2,40 & 3,70 & 2,60 \\
\hline & $1 \times 10^{-5}$ & 3,50 & 3,40 & 3,40 & 3,40 & 3,70 & 3,60 \\
\hline & $1 \times 10^{-4}$ & 3,40 & 3,40 & 3,30 & 3,40 & 3,50 & 3,60 \\
\hline & $1 \times 10^{-3}$ & 3,40 & 3,40 & 3,20 & 3,20 & 4,00 & 3,60 \\
\hline
\end{tabular}

$\mathrm{H}_{\text {dreno }}=$ coluna de água no interior do canal

$\mathrm{K}_{\text {terreno }}=$ condutividade hidráulica do terreno

$\mathrm{T}_{\text {trânsito }}=$ tempo de trânsito

Comparando-se as curvas para as quatro alturas de dreno para M1, M2 e M3, observou-se que a $\mathrm{P}_{\text {captura }}$ máxima foi em M3, cuja barreira foi mais efetiva que aquela colocada no modelo M2, com profundidade de $2 \mathrm{~m}$ (Figura 4).

Analisando-se o comportamento dessas curvas, notou-se que na ausência de recarga não houve evidência de que a profundidade de captura dependesse da condutividade hidráulica, dada a pequena variação nos valores de $\mathrm{P}_{\text {captura }}$ nos três modelos. Este comportamento, em situação de recarga, foi de modo geral diferente, com as curvas apresentando uma tendência em arco.

Em relação às larguras horizontais de captura das partículas ( $\mathrm{L}_{\text {captura }}$ ), os valores foram de 12,30 m a 17,40 m, conforme a Tabela 6. 
Tabela 6- Variação na largura horizontal de captura das partículas para M1, M2 e M3

Table 6- Horizontal width variation of the capture zone for M1, M2 e M3 models

\begin{tabular}{|c|c|c|c|c|c|c|c|}
\hline \multirow{4}{*}{$\mathbf{H}_{\text {dreno }}(\mathbf{m})$} & \multirow{4}{*}{$\begin{array}{c}\mathbf{K}_{\text {terreno }} \\
(\mathbf{m} / \mathbf{s})\end{array}$} & \multicolumn{6}{|c|}{ Modelo } \\
\hline & & \multicolumn{2}{|c|}{ M1 } & \multicolumn{2}{|c|}{ M2 } & \multicolumn{2}{|c|}{ M3 } \\
\hline & & \multicolumn{2}{|c|}{$\mathbf{L}_{\text {captura }}(\mathbf{m})$} & \multicolumn{2}{|c|}{$\mathbf{L}_{\text {captura }}(\mathbf{m})$} & \multicolumn{2}{|c|}{$\mathbf{L}_{\text {captura }}(\mathbf{m})$} \\
\hline & & $\begin{array}{c}\text { Sem } \\
\text { recarga }\end{array}$ & $\begin{array}{c}\text { Com } \\
\text { recarga }\end{array}$ & $\begin{array}{c}\text { Sem } \\
\text { recarga }\end{array}$ & $\begin{array}{c}\text { Com } \\
\text { recarga }\end{array}$ & $\begin{array}{c}\text { Sem } \\
\text { recarga }\end{array}$ & $\begin{array}{c}\text { Com } \\
\text { recarga }\end{array}$ \\
\hline \multirow{4}{*}{1,00} & $1 \times 10^{-6}$ & 12,70 & 13,90 & 16,10 & 17,00 & 15,80 & 16,40 \\
\hline & $1 \times 10^{-5}$ & 12,80 & 12,90 & 16,30 & 16,70 & 15,70 & 15,80 \\
\hline & $1 \times 10^{-4}$ & 12,70 & 12,70 & 16,10 & 15,00 & 15,80 & 15,70 \\
\hline & $1 \times 10^{-3}$ & 12,70 & 12,30 & 15,30 & 16,40 & 15,80 & 15,50 \\
\hline \multirow{4}{*}{0,75} & $1 \times 10^{-6}$ & 15,20 & 16,30 & 17,20 & 17,10 & 16,80 & 17,40 \\
\hline & $1 \times 10^{-5}$ & 15,50 & 15,50 & 17,30 & 17,30 & 17,00 & 17,00 \\
\hline & $1 \times 10^{-4}$ & 15,50 & 15,30 & 17,10 & 17,20 & 17,00 & 16,90 \\
\hline & $1 \times 10^{-3}$ & 14,60 & 14,60 & 16,80 & 16,80 & 17,00 & 16,60 \\
\hline \multirow{4}{*}{0,50} & $1 \times 10^{-6}$ & 14,50 & 15,00 & 16,50 & 17,00 & 16,10 & 16,60 \\
\hline & $1 \times 10^{-5}$ & 14,30 & 14,50 & 16,50 & 16,50 & 16,00 & 16,00 \\
\hline & $1 \times 10^{-4}$ & 14,40 & 14,20 & 16,40 & 16,50 & 16,00 & 16,00 \\
\hline & $1 \times 10^{-3}$ & 15,80 & 15,90 & 17,50 & 17,50 & 16,00 & 17,20 \\
\hline \multirow{4}{*}{0,30} & $1 \times 10^{-6}$ & 15,40 & 15,70 & 17,00 & 17,30 & 16,50 & 17,10 \\
\hline & $1 \times 10^{-5}$ & 15,40 & 15,20 & 16,80 & 16,00 & 16,40 & 16,50 \\
\hline & $1 \times 10^{-4}$ & 15,00 & 14,80 & 16,50 & 15,70 & 16,30 & 16,30 \\
\hline & $1 \times 10^{-3}$ & 14,50 & 14,40 & 16,40 & 15,30 & 16,20 & 16,20 \\
\hline
\end{tabular}

$\mathrm{H}_{\text {dreno }}=$ coluna de água no interior do canal

$\mathrm{K}_{\text {terreno }}=$ condutividade hidráulica do terreno

$\mathrm{T}_{\text {trânsito }}=$ tempo de trânsito

De acordo com os valores desta tabela, os melhores resultados foram para $\mathrm{H}_{\text {dreno }}=0,75 \mathrm{~m} \mathrm{e}$ os piores para $\mathrm{H}_{\text {dreno }}=1 \mathrm{~m}$. A largura de captura aumentou com a inserção da barreira em forma de cone (M2 e M3), mostrando que esta barreira foi mais efetiva que a vertical, descrita no item anterior, pois aumentou $\mathrm{L}_{\text {captura }}$ entre $2 \mathrm{~m} \mathrm{e} 4 \mathrm{~m}$. Não foram observados efeitos significativos da recarga neste parâmetro.

Também as variações com a mudança de condutividade hidráulica para cada $\mathrm{H}_{\text {dreno }}$ não foram superiores a $1 \mathrm{~m}$ (M1). Em M2 e M3 os valores foram bem próximos para os diferentes $\mathrm{H}_{\text {drenos. }}$

Os gráficos da figura 11 ilustram as relações de $\mathrm{P}_{\text {captura }}$ e $\mathrm{L}_{\text {captura }}$ em função de $\mathrm{K}_{\text {terreno }}$ para os diferentes $\mathrm{H}_{\text {dreno. }}$.
Uma vez que os resultados de M1, M2 e M3 não apresentaram uma eficiência satisfatória, na tentativa de se obter como resultado uma vazão de dreno superior a $10 \mathrm{~m}^{3} / \mathrm{dia}$, para um tempo de trânsito entre 10 e 15 dias, foi construído o modelo M4 (Figura 12). A geometria e a área da wetland foram ajustadas com o objetivo de atingir o melhor resultado dentro dos limites impostos, o que foi alcançado com uma $\mathrm{Q}_{\text {dreno }}$ de $16,28 \mathrm{~m}^{3} /$ dia e um t trânsito mínimo de 10 dias e máximo de 12 dias, para um $\mathrm{H}_{\text {dreno }}$ de $0,9 \mathrm{~m}$ nas saídas dos canais de fluxo subsuperficial e $\mathrm{K}_{\text {terreno }}$ igual a $1 \times 10^{-5} \mathrm{~m} / \mathrm{s}$, e uma área pouco superior a $2000 \mathrm{~m}^{2}$. 


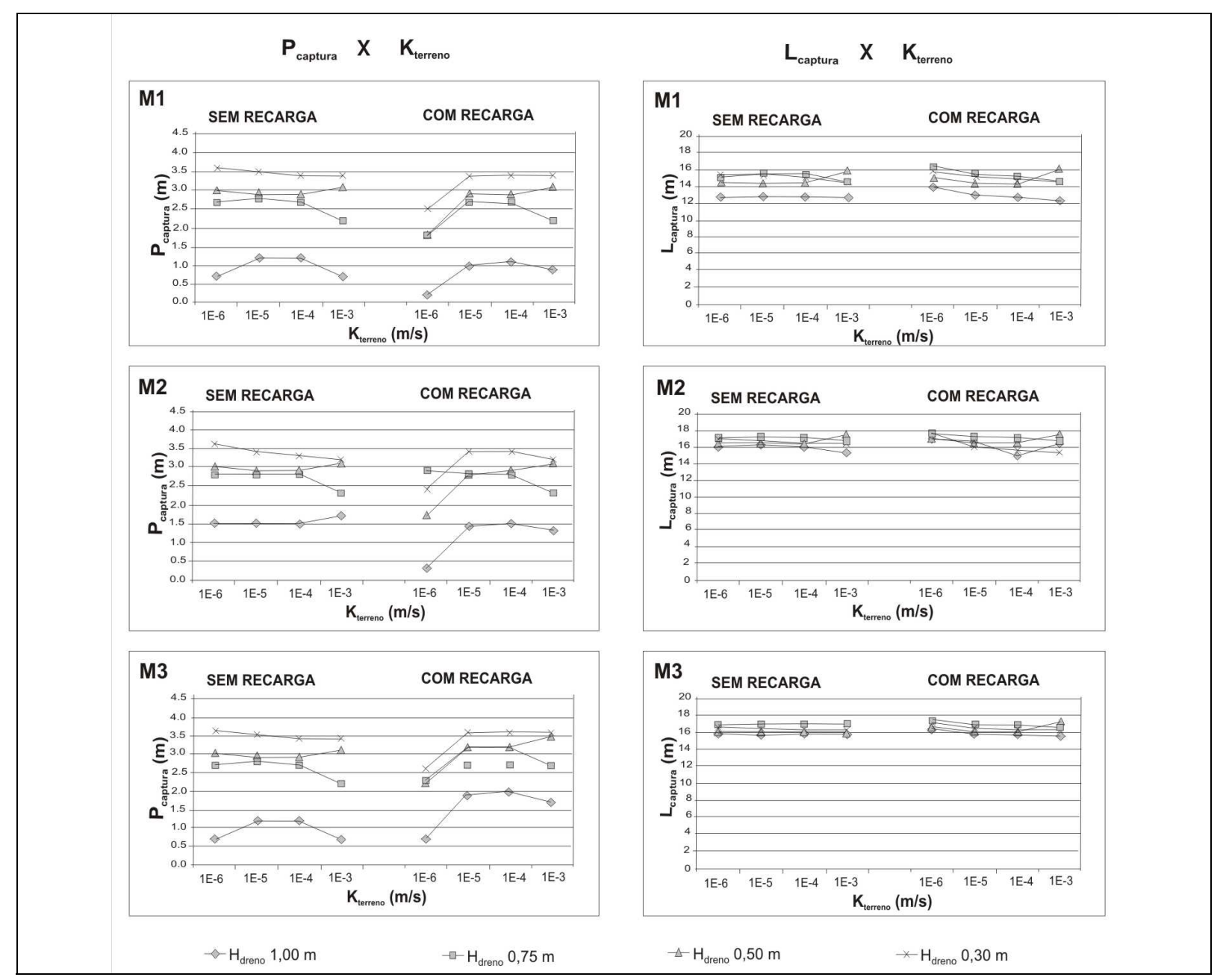

Figura 11- Profundidades e Larguras de captura das partículas para os modelos M1, M2 e M3, respectivamente Figure 11- Depth and width of the capture zone of the particles for the M1, M2 and M3 models, respectively

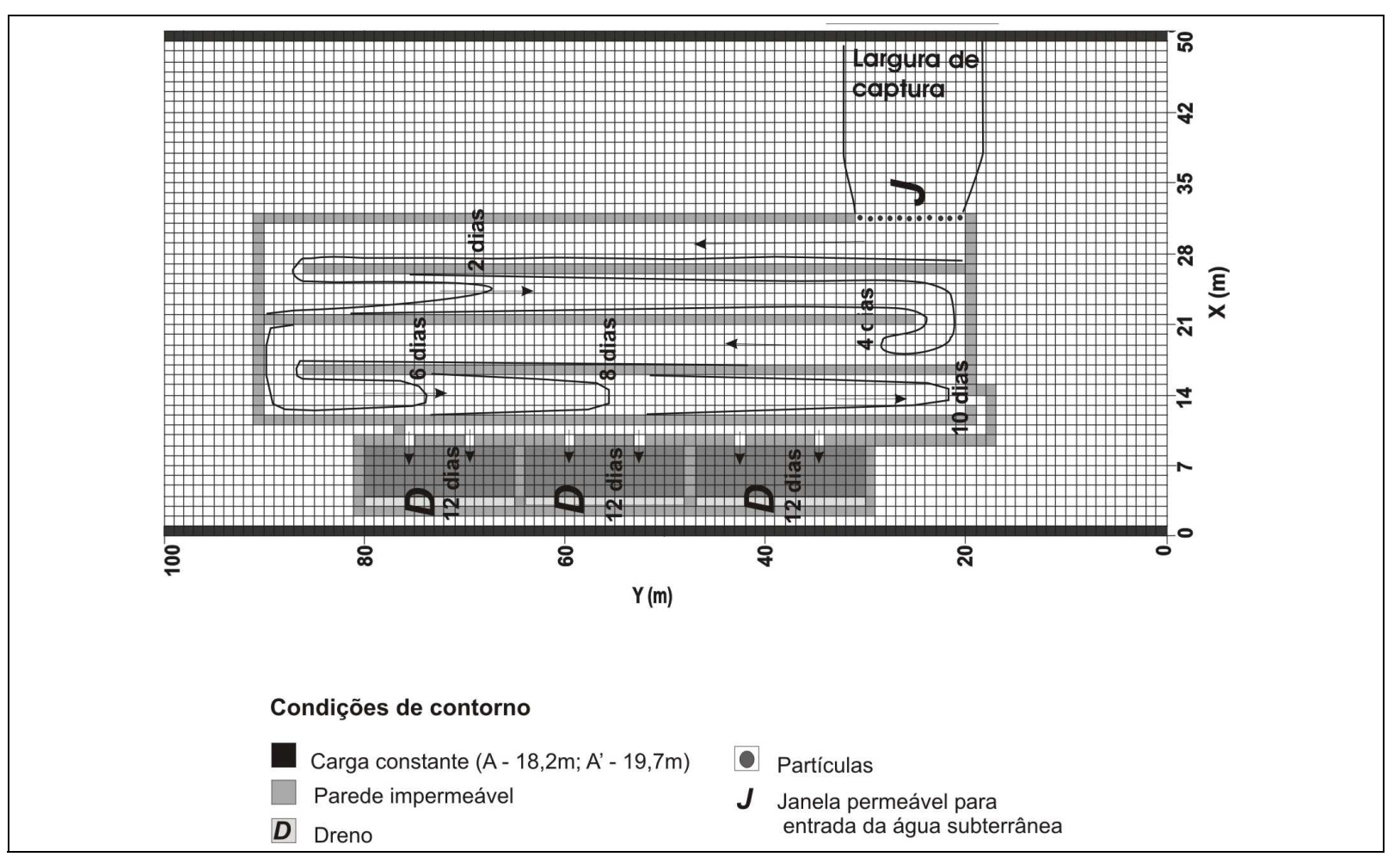

Figura 12- Exemplo de resultado da trajetória das partículas para o modelo M4

Figure 12- Example of the results of particle trajectory for the model $M 4$ 


\section{CONCLUSÕES}

A utilização do Visual Modflow para a simulação de wetlands construídas para captura de plumas contaminantes em aqǘferos livres e rasos se mostrou viável. Porém, os resultados indicaram a necessidade de uma área considerável $\left(>2000 \mathrm{~m}^{2}\right)$ para a implantação desses sistemas, para tratar água subterrânea a partir de $10 \mathrm{~m}^{3} /$ dia, com um tempo de residência entre 10 e 15 dias.

A vazão drenada e o tempo de permanência da água dentro do canal são inversamente proporcionais entre si e fortemente controlados pela condutividade hidráulica do terreno $\left(\mathrm{K}_{\text {terreno }}\right)$, que é um fator limitante para a construção de wetland para tratar a água subterrânea. Para
$\mathrm{K}_{\text {terreno }} \leq 1 \times 10^{-6} \mathrm{~m} / \mathrm{s}$, o tempo de residência seria muito longo e o volume drenado muito pequeno, inferior a $1 \mathrm{~m}^{3} /$ dia.

A precipitação também influencia o tempo de trânsito. Nas simulações com recarga de $250 \mathrm{~mm} /$ ano esse tempo foi menor que nas simulações sem recarga.

A largura da pluma capturada pode ser controlada com barreiras impermeáveis para aumentar a abertura de captura da água subterrânea. Já a profundidade da pluma exige uma intervenção mais complexa, pois barreiras de até $4 \mathrm{~m}$ além do fundo do canal aumentariam, no máximo, até $1 \mathrm{~m}$ a profundidade de captura da água subterrânea. 


\section{REFERÊNCIAS}

BRADLEY, C. Transient modeling of water-table variation in a floodplain wetland, Narborough Bog, Leicestershire. Journal of Hydrology. N. 185, p 87114, 1996

FREEZY, R. \& CHERRY, J. Groundwater. New Jersey, Prentice-Hall, 1979

GUIGUER, N. \& FRANZ, T. Visual MODFLOW. User's manual. Waterloo, WHI, 1996.

HENSEL, B. R. \& MILLER, M. V. Effects of wetlands creation on groundwater flow. Journal of Hydrology. N. 126, p 293-314, 1991.

KADLEC, R. H. \& KNIGHT, R. L. Treatment Wetlands. Lewis Publishers, 1996.

RESTREPO, J. I.; MONTOYA, A. M.; OBEYSEKERA, J. A wetland simulation for the MODFLOW ground water model. Groundwater. N 36 (5), p 764-770, 1998.

SALATI, E.; SALATI E., SALATI FILHO, E. Project of wetlands developed in Brazil. In: $6^{\text {th }}$ INTERNATIONAL CONFERENCE ON WETLANDS SYSTEMS FOR WATER POLLUTION CONTROL, Águas de São Pedro, SP, Brasil. p 11-27, 1998.

TCHOBANOGLOUS, G. Constructed wetlands and aquatic plant systems: research, design, operational, and monitoring issues. In: MOSHIRI, G. A. (Ed), Constructed wetlands for water quality improvement. Lewis Publishers, p 23-33, 1993.

\section{AGRADECIMENTOS}

Os autores gostariam de agradecer à FAPESP pelo auxílio financeiro a este trabalho (processo $n^{\circ}$. 97/6950-6), e à CAPES, pela bolsa de estudos à primeira autora. 